\title{
The Value of Fundoscopy in General Practice
}

\author{
Irini P. Chatziralli ${ }^{*}, 1,2$, Evgenia D. Kanonidou ${ }^{1}$, Petros Keryttopoulos ${ }^{3}$, Prodromos Dimitriadis ${ }^{3}$ and \\ Leonidas E. Papazisis ${ }^{1}$
}

\author{
${ }^{I}$ Department of Ophthalmology, General Hospital of Veroia, Veroia, Greece \\ ${ }^{2}$ Eye Department, Barts and the London NHS Trust, London, UK \\ ${ }^{3}$ Department of Internal Medicine, General Hospital of Veroia, Veroia, Greece
}

\begin{abstract}
Background: Many of the common systemic diseases present characteristic changes in the fundus of the eye, but fundoscopy is often performed by an ophthalmologist. Our purpose was to assess the value of fundoscopy for the general practitioners (GPs) regarding the diagnosis and management of the cases which they face in daily practice.

Methods: 689 patients were referred by GPs to the outpatient ophthalmology department for fundoscopy during the year 2010. The causes of this referral, the results of ophthalmoscopy and its significance in the final diagnosis were recorded and analyzed.

Results: In 22 patients (3.1\%), fundoscopy revealed optic disc edema. In 7 patients with head trauma (9.7\%), fundoscopy revealed intravitreous haemorrhage and Berlin edema. From the patients with photopsias or floaters, $5(10.2 \%)$ had retinal detachment. Finally, in cases with diabetes mellitus or hypertension, ophthalmoscopy was very important to detect the existence and grade the degree of diabetic or hypertensive retinopathy, if they appeared, and as a result to evaluate the prognosis of the disease.

Conclusions: Fundoscopy is fundamental for the GP, as it may help to confirm or exclude the diagnosis of many common diseases. Nevertheless, there are clinical entities where ophthalmoscopy should be performed by an ophthalmologist, in order to be more specific and accurate, and GP should be able to recognise these cases.
\end{abstract}

Keywords: Fundoscopy, general practice, ophthalmoscopy.

\section{INTRODUCTION}

Many of the common systemic diseases present characteristic changes in the fundus of the eye. These changes can be observed before the clinical manifestations of the disease, which come at a later stage. Consequently, the value of fundoscopy has been well established and every medical doctor is expected to be able to use the ophthalmoscope [1-3]. However, in the majority of public hospitals, fundoscopy is performed by an ophthalmologist, even though it may concern cases of general practice. Under the light of the above, this study aims to assess the value of fundoscopy for the general practitioners (GPs) regarding the diagnosis and management of the cases which they face in daily practice.

\section{MATERIALS AND METHODOLOGY}

Participants in our study were 689 patients (297 male and 392 female), age range from 7 to 78 years old (median age 38 years old), who were referred by GPs to the Ophthalmology Outpatient Department at General Hospital of Veroia, Greece for fundoscopy during the year 2009. Specifically, from the total number of referred cases, 517

*Address correspondence to this author at the 28 Papanastasiou street, Agios Dimitrios, Athens, 17342, Greece;

Tel: 00306973046326; Fax: 00302109312008;

E-mails: eirchat@yahoo.gr, Irini.Chatziralli@bartsandthelondon.nhs.uk patients were referred by the emergency department due to headache (27.5\%-142 of 517 patients), dizziness or vertigo (8.7\%-45 of 517), fever of unknown origin (FUO) $(32.7 \%$ 169 of 517), head trauma (13.9\%-72 of 517), photopsias or floaters $(9.4 \%-49$ of 517$)$, hypertension crisis $(2.3 \%-12$ of $517)$ and acute stroke (5.5\%-28 of 517). 172 patients were also referred by the internal medicine department to investigate if their eyes were affected by diabetes mellitus (56.9\%-98 of 172) or hypertension (43.1\%-74 of 172). All patients underwent dilated fundoscopy by an ophthalmologist, using the indirect ophthalmoscope. The outcome of ophthalmoscopy was recorded in each case.

\section{RESULTS}

In 6 patients with headache (4.2\%), the fundoscopy revealed edema of the optic disc caused by an endocranial tumor, as it was confirmed later by computerized tomography. Fundoscopy in all patients suffered from dizziness or vertigo was normal, as well as in those with hypertension crisis. In 9 patients with FUO (5.3\%), ophthalmoscopy contributed to the diagnosis of a central nervous system (CNS) infection i.e., meningitis. Moreover, it was also necessary for the other patients with FUO, as it was considered mandatory before performing a lumbar puncture to evaluate the possibility of CNS infection. In 7 patients with head trauma (9.7\%), fundoscopy revealed intravitreous haemorrhage and Berlin edema, while in 4 patients (5.6\%) it showed signs of increased intracranial pressure due to 
subarachnoid haemorrhage. Increased intracranial pressure was also found in 3 patients with acute stroke (10.7\%), in which papilloedema was observed in fundoscopy. From the patients with photopsias or floaters, $5(10.2 \%)$ had retinal detachment, while $5(10.2 \%)$ had retinal breaks. Finally, in cases with diabetes mellitus or hypertension, ophthalmoscopy was very important to detect the existence and grade of diabetic or hypertensive retinopathy, if they appeared, and as a result to evaluate the prognosis of the disease. It is also worthy to mention that in 8 from the total number of the 689 cases $(1.2 \%)$ glaucoma has been first diagnosed by the damage of the optic nerve observed via fundoscopy.

\section{DISCUSSION}

Usually GPs and doctors of internal medicine refer patients to ophthalmologists to undergo fundoscopy either as an "emergency" case or in the context of a detailed systemic clinical examination. In "emergency" cases, the most frequent question is whether optic disc edema exists. Papilloedema is optic disc swelling secondary to increased intracranial pressure and its presence should raise the suspicion of serious intracranial pathology; ie. tumors, venous sinus thrombosis, subarachnoid haemorrage, CNS infection, haematoma or idiopathic intracranial hypertension. In these cases, ophthalmoscopy is a very important diagnostic tool in GP's hands. Fundoscopy's value is also significant in cases of head trauma, as it can reveal intracranial haemorrhages or causes of low vision (intravitreous haemorrhage). The GP should refer the patient to an expert doctor (neurosurgeon or ophthalmologist respectively) for further investigation and management of these cases.

Another special finding in fundoscopy is retinal or vitreous detachment, something difficult for a GP to diagnose with certainty. In these cases, the GP should be suspicious from the history of the patient and from the presented signs and symptoms, such as photopsias or floaters. If the GP is not sure about the diagnosis of retinal breaks or retinal detachment, it is better to refer the patient to an ophthalmologist, because this is an ocular emergency and may cause loss of vision, when treatment is not appropriate.

The fundus of the eye may reveal changes related to common diseases such as hypertension and diabetes mellitus [1-4]. In a large percentage of patients with hypertension, sclerotic changes are primarily seen in the choroidal vessels and later exudation and flame shaped haemorrhages give the picture of hypertensive retinopathy. In addition to this, diabetic retinopathy usually appears in elderly people, having diabetes mellitus for 10 years or more and may be proliferative (neovascularization, vitrous haemorrhages or vitrous detachment) or non-proliferative (retinal microaneurysms, exudates, haemorrhages) [5]. The GP should be able to recognise pathological changes in the fundus concerning the aforementioned diseases and if he finds something suspicious, he should always refer the patient to an ophthalmologist for a more specific and detailed ophthalmological examination, but not as an "emergency" case [5].

It is worthy to mention that "unnecessary" referrals could be reduced by telemedicine, which uses the combination of telecommunication and information technologies to provide and support healthcare [6]. As a part of telemedicine, teleophthalmology provides eye consultation by delivering high-quality eye images and videos over a public broadband network [7]. In this way, GPs could have a database of retina images to compare with the image found in fundoscopy, so as to identify which case is really "urgent". In addition, they could seek advice from an ophthalmologist by telemedicine program, therefore the ophthalmology outpatients' department would not be burdened with "unnecessary" referrals.

\section{CONCLUSION}

The study demonstrates that fundoscopy is an essential skill for the GP and should not be underestimated, as it may be necessary to confirm or exclude the diagnosis of many common ocular or systemic diseases. However, there are clinical entities where ophthalmoscopy should be performed by an ophthalmologist, in order to obtain a more specific and accurate diagnosis, and the GP should be able to recognise these cases. Further examination of the eye with fundoscopy can assist the diagnosis of systemic disease.

\section{ACKNOWLEDGEMENT}

None declared.

\section{CONFLICT OF INTEREST}

None declared.

\section{REFERENCES}

[1] Gilkes MJ. The GP and the specialist ophthalmology. Br Med J 1982; 285: 1247-8.

[2] Gurland BB. The GP and the ophthalmoscope. J Med Soc N J 1964; 61: 447-8.

[3] Ganguly AK. Scope of ophthalmoscopy in general practice and its significance. Indian Med J 1964; 61:39-41.

[4] Griffith SP, Freeman WL, Shaw CJ, et al. Screening for diabetic retinopathy in a clinical setting: a comparison of direct ophthalmoscopy by primary care physicians with fundus photography. J Fam Pract 1993; 37: 49-56.

[5] Gill JM, Cole DM, Lebowitz HM, Diamond JJ. Accuracy of screening for diabetic retinopathy by family physicians. Ann Fam Med 2004; 2: 218-20.

[6] Li HK. Telemedicine and ophthalmology. Surv Ophthalmol 1999; 44: 61-72.

[7] Tang RA, Morales M, Ricur G, Schiffman JS. Telemedicine for eye care. J Telemed Telecare 2005; 11:391-6. 